\title{
Myocardial perfusion SPECT findings in postCOVID period
}

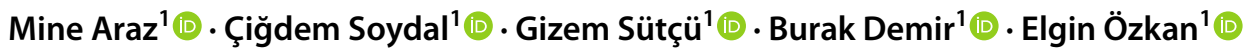

Received: 3 August 2021 / Accepted: 29 August 2021 / Published online: 7 September 2021

(c) The Author(s), under exclusive licence to Springer-Verlag GmbH Germany, part of Springer Nature 2021

\begin{abstract}
Purpose To investigate if (i) the risk of ischemia on myocardial perfusion scan (MPS), (ii) number of coronary angiographies (CAG) performed, and (iii) necessity for invasive (stent implantation or coronary artery bypass grafting (CABG)) or medical treatment increased in patients infected with COVID-19.

Methods Patients who were referred to MPS between August 2020 and April 2021 with a history of active symptomatic COVID-19 infection (confirmed by PCR positivity) in the last 6 months were involved in the study group. Age-and gendermatched control group was composed of randomly chosen patients who attended for MPS between January 2019 and September 2019, before pandemic. Frequency of ischemia, CAG, and invasive or medical treatments were compared between groups. Results Ischemia was reported more frequently in the study group $(p<0.001)$. In clinical evaluation, regardless of the MPS results, the necessity for invasive evaluation with CAG and treatment (either medical therapy or invasive interventions) was higher in the study group $(p=0.006$ and $p=0.015)$. It was also true for patients with abnormal MPS results $(p=0.008$ and $p=0.024)$ but not for the patients with ischemia $(p=0.29$ and $p=0.06)$.

Conclusion There exists a significant increase in the frequency of ischemia on MPS, undergoing CAG, stent implantation or CABG, and initiation of medical therapy in patients with a history of COVID-19 infection in the last 6 months. MPS is a reliable method in patients who present with cardiovascular symptoms in the late COVID period.
\end{abstract}

Keywords COVID-19 $\cdot$ Radionuclide imaging $\cdot$ Perfusion $\cdot$ Ischemia

\section{Introduction}

COVID-19 has affected healthcare systems and changed paradigms in the whole world in the last 1 year. Different from other upper respiratory tract viruses, COVID-19 appears to act on many systems in the body, inducing the inflammatory cascade and thrombotic process. It has been reported that risk of cardiac events has increased after COVID-19 infection [1]. Sudden cardiac death is also reported among young patients, which is soon related with late thrombotic complications. Researchers have demonstrated that there was a significant increase in hospital admission rates due to acute myocardial infarction in the late phase of the first wave of the pandemic, compared to rates before pandemic [2].

This article is part of the Topical Collection on Cardiology

Mine Araz

minesoylu@yahoo.com

1 Department of Nuclear Medicine, Ankara University Medical School, Ankara, Turkey
Myocardial perfusion scan is the fundamental functional imaging method in evaluation of ischemia and coronary risk assessment. During the pandemic, there have been several reports demonstrating the steep decline in the number of myocardial perfusion studies performed in nuclear medicine departments [3]. However, its use in patients infected with COVID-19 has not been presented yet.

In this study, we investigated if (i) the risk of ischemia on MPS, (ii) number of coronary angiographies performed, and (iii) necessity for invasive (stent implantation or coronary artery bypass grafting (CABG)) or medical treatment increased in patients infected with COVID-19.

\section{Materials-methods}

\section{Patients}

Following ethics committee approval (2021/117) for this retrospectively designed study, patients who were referred to the nuclear medicine department between August 2020 
and April 2021 with a history of active symptomatic COVID-19 infection in 6 last months were involved in the study group. Age- and gender-matched control group was composed of randomly chosen patients without history of COVID-19 history who attended for MPS between January 2019 and September 2019, before pandemic. Age, gender, risk factors for coronary artery disease (hypertension, diabetes mellitus, hyperlipidemia, family history for coronary artery disease, obesity), as well as the active symptoms the patients were investigated for were noted in both groups. The history of coronary stent or CABG was also recorded from the hospital registry. Myocardial perfusion SPECT results were categorized in the groups as either normal or abnormal. Abnormal results were then regrouped as (i) ischemia either together with infarct or not and (ii) infarct only.

\section{Myocardial perfusion SPECT}

Myocardial perfusion gated-SPECT study was performed by physical or pharmacological stress tests, following recommendations of the European Association of Nuclear Medicine [4]. During pandemic, additional precautions were taken in the nuclear cardiology facility in order to protect both patients and the staff according to previously published statements. Pharmacological stress test was preferred over exercise stress testing due to higher droplet exposure risk in exercise test. However, regadenoson is not available, and adenosine is the only choice. Considering the side effects of adenosine, change in ordered stress protocol was made cautiously, and pharmacological stress could be performed only to patients who were undoubtedly eligible for adenosine stress. In conditions where exercise testing was determined to be necessary, higher-level PPE, face shield, and gloves were used by the staff and surgical mask or face covering by the patients. Extra time was also allowed in order to clean the room [5].

Stress-rest 2-day protocol was followed. 350-700 MBq Tc-99 m methoxyisobutylisonitrate (MIBI) was intravenously administered on 2 following days, both for stress and rest imaging.

\section{Stress protocols}

For pharmacological stress test, intravenous adenosine was used. $140 \mu \mathrm{g} / \mathrm{kg} / \mathrm{min}$ adenosine was infused over $6 \mathrm{~min}$. Bolus radiopharmaceutical injection was performed on 3rd minute of infusion. For treadmill exercise stress test, Bruce protocol was followed. Radiopharmaceutical injection was performed at at least $85 \%$ of age-adjusted maximum predicted heart rate.

\section{Imaging protocol}

Dual head gamma camera equipped with low energy-high resolution collimator (DDD CorCam, Horsholm, Denmark) was used. Stress SPECT images were obtained $45 \mathrm{~min}$ after radiopharmaceutical injection. Stress SPECT images were acquired over $180^{\circ}$ angular sample range, $20 \mathrm{~s} /$ rotation, and a total of 64 views from right anterior oblique to left posterior oblique position with detectors positioned at $90^{\circ}$ angle, with a non-circular orbit. Rest gated-SPECT study was performed $60 \mathrm{~min}$ later than the radiopharmaceutical administration with the same parameters of acquisition. 8-frame gating was used. A beat length acceptance window of $20 \%$ was set. Data were recorded in $64 \times 64$ matrix.

\section{Image interpretation}

Areas of diminished perfusion were analysed with a software programme (Corridor4DM) visually and quantitatively. Left ventricular ejection fraction (LVEF) and wall motion were examined from gated images. An even distribution of Tc-99 m MIBI in the left ventricle together with a normal LVEF and wall motion is considered normal. An abnormal MPS was defined as existence of abnormal stress perfusion either resolved (ischemia) or remained constant (infarct) in rest images. Abnormal LVEF and/or wall motion was also interpreted as an abnormal scan [4].

\section{Statistics}

Descriptive statistics were presented as mean \pm standard deviation for continuous variables and percentage for categorical variables. Categorical variables were expressed as frequencies (percentage) and compared using the Pearson chi-square test. Student's $t$-test was performed to compare means and chi-square test for comparison of categorical variables. $P$ values $<0.05$ were considered significant. SPSS version 25 was used for statistical analysis.

\section{Results}

A total of 179 patients $(84 \mathrm{~F}, 95 \mathrm{M}$, mean age $58.28 \pm 9.28)$ were involved retrospectively. Eighty-five patients were included in the study group and 94 in the control group. Mean interval between COVID-19 PCR positivity and MPS was $90.26 \pm 47.08$ days (min, 16 days; max, 180 days). Adenosine and exercise stress was performed in $59(69.4 \%)$ and $26(30.5 \%)$ in the study group and in $61(64.8 \%)$ and $33(35.1 \%)$ patients in the control group, respectively $(p=0.52)$. The study and control groups were matched in terms of age and sex. No significant differences were found in the frequency of risk factors and previous 
Table 1 Demographics and clinical characteristics of patients in the study and control groups

\begin{tabular}{lllr}
\hline & $\begin{array}{l}\text { Study group } \\
\text { Mean } \pm \text { SD }\end{array}$ & $\begin{array}{l}\text { Control group } \\
\text { Mean } \pm \text { SD }\end{array}$ & $P$ value \\
\hline Age & $58.13 \pm 9.07$ & $58.43 \pm 9.42$ & 0.98 \\
& $n(\%)$ & $n(\%)$ & \\
Male gender & $43(50.5)$ & $52(55.3)$ & 0.55 \\
Hypertension & $59(69.4)$ & $54(57.4)$ & 0.07 \\
Diabetes & $33(38.8)$ & $36(38.2)$ & 0.53 \\
Hyperlipidemia & $42(49.4)$ & $43(45.7)$ & 0.37 \\
Prior history of CABG & $9(9.5)$ & $3(3.5)$ & 0.09 \\
Prior history of stent & $27(28.7)$ & $15(17.6)$ & 0.06 \\
Family history & $40(47)$ & $52(57.1$ & 0.23 \\
Smoking & $35(41.1)$ & $48(52.1)$ & 0.30 \\
Obesity & $32(37.6)$ & $40(42.5)$ & 0.27 \\
Chest pain & $48(56.4)$ & $48(51)$ & 0.28 \\
Dispnea & $35(41.1)$ & $13(13.8)$ & $<0.001$ \\
\hline
\end{tabular}

Table 2 Distribution of frequencies of myocardial perfusion scintigraphy results among the study and control groups

\begin{tabular}{lllr}
\hline & $\begin{array}{l}\text { Study group } \\
n(\%)\end{array}$ & $\begin{array}{l}\text { Control group } \\
n(\%)\end{array}$ & $P$ value \\
\hline Abnormal MPS & $46(48.9)$ & $39(54.1)$ & 0.09 \\
İschemia & $39(45.8)$ & $19(20.2)$ & $<0.001$ \\
\hline
\end{tabular}

coronary interventions or operations for coronary artery disease between two groups (Table 1).

In the analysis of the dominant symptom for referral to MPS, dispnea was apparently more common among the patients in the study group compared to the control group $(p<0.001)$, but the frequency of chest pain did not differ among the groups $(p=0.28)$ (Table 1). Myocardial perfusion scan was abnormal in 85 patients (45.5\%). Ischemia was reported more frequently among patients with a history of COVID-19 infection ( $\mathrm{p}<0.001)$ (Table 2).

When it comes to clinical evaluation, regardless of the MPS results, the necessity for invasive evaluation with coronary angiography and treatment (either medical therapy or invasive interventions, that is, stent implantation or CABG) was higher in the study group ( $p=0.006$ and $p=0.015$, respectively). The same was true for patients with abnormal MPS results ( $p=0.008$ and $p=0.024$ ) but not for the patients with MPS result ischemia $(p=0.29$ and $p=0.06$, respectively) (Table 3 ).

Among 39 patients with ischemia on MPS in the study group, 11 patients were taken under follow-up without coronary angiography, and medical therapy was started. Five patients refused coronary angiography, and 23 patients were evaluated with coronary angiography. Stent implantation was performed in $2 / 23$ patients and CABG in 1 patient, and in 20/23 patients, medical therapy was preferred due to noncritical stenosis in the coronaries. One of the patients with stent implantation had no previous history of coronary artery disease or any risk factors.

\section{Discussion}

COVID-19 has appeared as a game changer in the world, affecting millions of people with a wide range of symptoms, as well as late complications, some of which are surprisingly frequent and life-threatening. Acute cardiac and vascular involvement in COVID-19 infection was reported in mostly hospitalized cases: myocarditis, acute coronary syndrome, pulmonary thromboembolism, cerebrovascular events, etc. Although acute coronary syndrome was relatively rare at initial presentation or during hospitalization, it could even affect patients with no obstructive coronary disease [6]. Endothelial dysfunction, progression of atheromatous plaque formation, emotional factors or hypoxia causing autonomous dysfunction, increased sympathetic vasomotor tonus, and diminished hemodynamics along with instability of the plaques caused by systemic inflammation were the pathophysiological mechanisms explained so far [7-9].

"Long-COVID" is the term referring to the clinical situation where signs or symptoms of acute COVID-19 have prolonged or new signs or symptoms developed over 12 weeks [10]. Chest pain was also defined as a possible component

Table 3 Follow-up results in control and study groups according to MPS results

\begin{tabular}{|c|c|c|c|c|}
\hline & & $\begin{array}{l}\text { Study group } \\
n(\%)\end{array}$ & $\begin{array}{l}\text { Control group } \\
n(\%)\end{array}$ & $P$ value \\
\hline \multirow[t]{2}{*}{ Any MPS result } & Necessity for $C A G$ & $33(38.8)$ & $19(20.2)$ & 0.006 \\
\hline & Treatment (medical, stent implantation, or $C A B G$ ) & $30(35.2)$ & $18(19.1)$ & 0.015 \\
\hline \multirow[t]{2}{*}{ Abnormal MPS result } & Necessity for CAG & $32(69.5)$ & $16(41.0)$ & 0.008 \\
\hline & Treatment (medical, stent implantation, or $C A B G$ ) & $29(63.0)$ & $15(38.4)$ & 0.024 \\
\hline \multirow[t]{2}{*}{ Ischemia on MPS } & Necessity for CAG after ischemia on MPS & $23(58.9)$ & $11(57.8)$ & 0.29 \\
\hline & Treatment (medical, stent implantation, or $C A B G$ ) & $27(58.6)$ & $15(38.4)$ & 0.06 \\
\hline
\end{tabular}


of "Long COVID", and many imaging studies have demonstrated that myocardial involvement existed in symptomatic patients in the late period [11-17].

Except from direct myocardial involvement, late coronary vascular events have also been demonstrated recently. A young female case has attended to hospital with chest pain 1 month later than recovery from severe respiratory syndrome caused by COVID-19. She had no previous history of cardiac disease or any known risk factors. Authors advocated that, as proven with adenosine stress cardiac myocardial MRI in this case, coronary microvascular ischaemia may have been the mechanism of persistent chest pain after recovery from COVID-19 [18]. Another case report presented a similar scenario on a young male patient, with gradually progressive dispnea on exercise. He was then diagnosed with acute cardiac failure due to nonobstructive coronary artery disease presenting with extensive myocardial infarction [19]. In our study, the indication of MPS was most commonly dispnea, but not chest pain. This finding actually points a gap in the literature. Dispnea may exist as a component of Long COVID in up to $40 \%$ of cases [20]. However, its relation to coronary vascular involvement is still unclear.

Myocardial perfusion scintigraphy is the most frequently used functional imaging tool for assessing obstructive coronary artery disease risk. There exists a small amount of articles studying myocardial perfusion scintigraphy in COVID19 patients.

Hasnie et al. have performed stress testing and myocardial perfusion imaging in 15 patients after recovery from COVID-19 and stated that both regadenoson and exercise stress tests were safe and stress myocardial perfusion scan can be used reliably in evaluation of ischemic heart disease after COVID-19 infection [21].

Hasnie et al. and Nappi et al. have hypothesized that the percentage of abnormal myocardial perfusion images would increase in the pandemic period, because those who have mild symptoms and a probably normal SPECT MPI would delay their examinations. However, no such significant increase was observed [3, 22].

The aim of this study was different than these previous reports; we focused only on patients with COVID-19 history and documented the increase in the incidence of ischemia demonstrable by MPS, compared to non-infected cohort. Although stent implantation was performed only in 2/39 and CABG in $1 / 39$ patients, ischemia was evident on myocardial perfusion scan in these patients. Keeping all other risk factors constant among the study and control group, COVID-19 has appeared as the possible reason for this difference in the frequency of ischemia between the study and the control group. Demonstration of ischemia reflects the functional changes in myocardial blood flow with stress, which supports the reported clinical cases and previously suggested possible pathophysiological mechanisms affecting myocardial blood flow after COVID-19 infection [6]. One patient with no previous history of coronary artery disease or any mentioned risk factors who come up with ischemia on MPS after recovery from COVID-19 is also meaningful considering the case reports mentioned above. The frequency of obstructive coronary disease indicating coronary interventions could be expected to be higher after COVID-19, concerning these microvascular endothelial changes, but it was found similar to the control group. Incidence of hospital admissions with acute MI was reported to be higher than the pre-pandemic period, but incidence of ischemia was not studied before [2]. Our results suggest that together with acute coronary syndromes, as a reflection of coronary dysfunction after recovery from COVID-19, ischemia on myocardial perfusion scan induced by stress testing is also more frequent compared to non-infected matched group of patients.

In clinical practice, MPS results guide patient evaluation by determining coronary artery risk. In our study, the number of coronary angiographies performed was higher in the study group. Initiation of medical therapy or invasive revascularisation therapies (stent implantation or $\mathrm{CABG}$ ) were also higher in patients after recovery from COVID-19. We believe that this finding deserves attention as there are no similar results reported before, keeping in mind that obviously we are still lacking of evidence to talk about a direct causal relationship between symptomatic COVID-19 infection and coronary artery disease. The reason why we could not demonstrate such an increase in patients with ischemia (a statistically nonsignificant increase in frequency of initiation of treatment still exists, with a $p=0.06$, Table 3 ), but generally in the whole study group regardless of the MPS results, may be the five patients in the ischemia group who did not accept to undergo CAG. Invasive evaluation regarding an abnormal MPS result other than ischemia may seem unnecessary anyway, but presence of any apparent perfusion defect, especially if developed recently, is probably a sign of relative functional disturbance in perfusion. If clinical risk factors are also worrisome, perfusion defects other than ischemia may also necessitate invasive evaluation, in order not to miss a pathology.

The main limitation of this study was its retrospective design. Loss of patient data and follow-up results led to a relative decrease in the number of subjects included. In order to reach a definite conclusion that COVID-19 is a cause or promoter for coronary artery disease, all patients should have been evaluated with CAG. Because this could not be maintained in a retrospective analysis, further prospective studies are necessary to confirm above mentioned results. Another thing is that COVID-19 is still a matter of debate with multiple unexplained issues in clinical practice. Together with vaccines and treatment methods, diagnosis 
and imaging remain an important area of clinical investigation to better understand and make provisions against late complications.

\section{Conclusion}

There is a significant increase in the frequency of ischemia on MPS in patients with a history of COVID-19 infection in the last 6 months. Frequency of undergoing coronary angiography, stent implantation or CABG, and initiation of medical therapy is also increased. MPS is a reliable method to investigate ischemia and guide management in patients who present with cardiovascular symptoms in the late COVID period.

Data availability Not applicable.

Code availability Not applicable.

\section{Declarations}

Ethics approval Ethics committee approval is taken (2021/117).

Conflict of interest The authors declare no competing interests.

\section{References}

1. Lala A, Johnson KW, Januzzi JL, Russak AJ, Paranjpe I, Richter F, Zhao S, Somani S, Van Vleck T, Vaid A, Chaudhry F, De Freitas JK, Fayad ZA, Pinney SP, Levin M, Charney A, Bagiella E, Narula J, Glicksberg BS, Nadkarni G, Mancini DM, Fuster V. Mount Sinai COVID Informatics Center. Prevalence and impact of myocardial injury in patients hospitalized with COVID-19 infection. J Am Coll Cardiol. 2020;76(5):533-46.

2. Fardman A, Oren D, Berkovitch A, Segev A, Levy Y, Beigel R, Matetzky S. Post COVID-19 acute myocardial infarction rebound. Can J Cardiol. 2020;36(11):1832.e15-1832.e16. https://doi.org/10. 1016/j.cjca.2020.08.016.

3. Nappi C, Megna R, Acampa W, Assante R, Zampella E, Gaudieri V, Mannarino T, Green R, Cantoni V, Petretta M, Cuocolo A. Effects of the COVID-19 pandemic on myocardial perfusion imaging for ischemic heart disease. Eur J Nucl Med Mol Imaging. 2021;48(2):421-7.

4. Verberne HJ, Acampa W, Anagnostopoulos C, Ballinger J, Bengel F, De Bondt P, Buechel RR, Cuocolo A, van Eck-Smit BL, Flotats A, Hacker M, Hindorf C, Kaufmann PA, Lindner O, Ljungberg M, Lonsdale M, Manrique A, Minarik D, Scholte AJ, Slart RH, Trägårdh E, de Wit TC, Hesse B. European Association of Nuclear Medicine EANM. EANM procedural guidelines for radionuclide myocardial perfusion imaging with SPECT and SPECT/CT 2015 revision. Eur J Nucl Med Mol Imaging. 2015;42(12):1929-40.

5. Skali H, Murthy VL, Paez D, Choi EM, Keng FYJ, Iain MA, Al-Mallah M, Campisi R, Bateman TM, Carrio I, Beanlands R, Calnon DA, Dilsizian V, Dondi M, Gimelli A, Pagnanelli R, Polk DM, Soman P, Einstein AJ, Dorbala S, Thompson RC. Guidance and best practices for reestablishment of non-emergent care in nuclear cardiology laboratories during the coronavirus disease 2019 (COVID-19) pandemic: an information statement from ASNC, IAEA, and SNMMI: Endorsed by the Infectious Diseases Society of America. J Nucl Cardiol. 2020;27(5):1855-62.

6. Lee CCE, Ali K, Connell D, Mordi IR, George J, Lang EM, Lang CC. COVID-19-associated cardiovascular complications. Diseases. 2021;9(3):47.

7. Ince, C; Mayeux, Philip R.; Nguyen, Trung; Gomez, Hernando; Kellum, John A.; Ospina-Tascón, Gustavo A.; Hernandez, Glenn; Murray, Patrick; De Backer, Daniel on behalf of the ADQI XIV Workgroup The Endothelium in Sepsis, SHOCK: March 2016 Volume 45 - Issue 3 - p 259-270

8. Libby P, Buring JE, Badimon L, Hansson GK, Deanfield J, Bittencourt MS, Tokgözoğlu L, Lewis EF. Atherosclerosis Nat Rev Dis Primers. 2019;5(1):56.

9. Feigl EO. Neural control of coronary blood flow. J Vasc Res. 1998;35(2):85-92.

10. National Institute for Health Research. Living with Covid-19. 2020. Available online: https://evidence.nihr.ac.uk/themedreview/ living-with-covid19

11. Sartoretti E, Sartoretti T, Imoberdorf R, Dracklé J, SartorettiSchefer S. Long-segment arterial cerebral vessel thrombosis after mild COVID-19. BMJ Case Rep. 2020;13:e236571.

12. Sollini M, Ciccarelli M, Cecconi M, Aghemo A, Morelli P, Gelardi F, Chiti A. Vasculitis changes in COVID-19 survivors with persistent symptoms: an [18F]FDG-PET/CT study. Eur J Nucl Med Mol Imaging. 2020;48:1460-6.

13. Jagia P, Ojha V, Naik N, Sharma S. Myocardial fibrosis detected by cardiovascular magnetic resonance in absence of myocardial oedema in a patient recovered from COVID 19. BMJ Case Rep. 2020;13:e240193.

14. Agarwal S, Al HH, Agarwal SK, Albastaki U. Possible association between myocardial infarction with nonobstructed coronary arteries and SARS-CoV-2 infection. CMAJ Can Med Assoc J. 2020;192:E1633-6.

15. Huang L, Zhao P, Tang D, Zhu T, Han R, Zhan C, Liu W, Zeng $\mathrm{H}, \mathrm{Tao} \mathrm{Q}, \mathrm{Xia}$ L. Cardiac involvement in patients recovered from COVID-2019 identified using magnetic resonance imaging. JACC Cardiovasc Imaging. 2020;13:2330-9.

16. Kotecha T, Knight DS, Razvi Y, Kumar K, Vimalesvaran K, Thornton G, Patel R, Chacko L, Brown JT, Coyle C, et al. Patterns of myocardial injury in recovered troponin-positive COVID-19 patients assessed by cardiovascular magnetic resonance. Eur Heart J. 2021;42:1866-78.

17. Puntmann VO, Carerj ML, Wieters I, Fahim M, Arendt C, Hoffmann J, Shchendrygina A, Escher F, Vasa-Nicotera M, Zeiher AM, et al. Outcomes of cardiovascular magnetic resonance imaging in patients recently recovered from coronavirus disease 2019 (COVID-19). JAMA Cardiol. 2020;5:1265.

18. Vallejo N, Teis A, Mateu L, Bayés-Genís A. Persistent chest pain after recovery of COVID 19 microvascular disease related angina. Eur Heart J Case Rep. 2021;5(3):ytab105. https://doi.org/10.1093/ ehjcr/ytab105.

19. Agarwal S, Al Hashimi H, Agarwal SK, Albastaki U. Possible association between myocardial infarction with nonobstructed coronary arteries and SARS-CoV-2 infection. CMAJ. 2020;192(48):1633-6.

20. Seeßle J, Waterboer T, Hippchen T, Simon J, Kirchner M, Lim A, Müller B, Merle U. Persistent symptoms in adult patients one year after COVID-19: a prospective cohort study. Clin Infect Dis. 2021 Jul 5:ciab611. https://doi.org/10.1093/cid/ciab611.

21. Hasnie UA, Hawi R, Andrikopoulou E, Iskandrian AE, Hage FG. Stress testing and myocardial perfusion imaging for patients after recovery from severe COVID-19 infection requiring hospitalization: a single-center experience. J Nucl Cardiol. 2021;26:1-7. 
22. Hasnie UA, Bhambhvani P, Iskandrian AE, Hage FG. Prevalence of abnormal SPECT myocardial perfusion imaging during the COVID-19 pandemic. Eur J Nucl Med Mol Imaging. 2021;48(8):2447-54.
Publisher's note Springer Nature remains neutral with regard to jurisdictional claims in published maps and institutional affiliations. 